\title{
Variation in Outcome in Tethered Cord Syndrome
}

\author{
Noorulain Iqbal, Mohsin Qadeer, Salman Yousuf Sharif \\ Department of Spinal and Neurosurgery, Liaquat National Hospital and Medical College, Karachi, Pakistan
}

Study Design: Fifty patients surgically treated for tethered cord syndrome (TCS) were retrospectively studied at Liaquat National Hospital, Karachi from 2010 until 2014.

Purpose: To assess the common presentations of TCS in our part of the world and the surgical outcome of the different presentations.

Overview of Literature: TCS is a stretch-induced functional disorder of the spinal cord with its caudal part anchored by an inelastic structure, which results in characteristic symptoms and signs. Due to the variety of lesions and clinical presentations and the absence of high-quality clinical outcome data, the decision regarding treatment is difficult.

Methods: Fifty consecutive patients with TCS were reviewed retrospectively with a follow-up period of 12-48 months. The majority of the patients were $0-15$ years of age with the mean age of 4 years. The presenting complaints and the associated pathologies were documented, and the patients were assessed using the new Karachi TCS severity scale for clinical assessment.

Results: Eighty five percent of the patients with thickened filum terminale improved. Sixty six percent of the patients with diastematomyelia, $60 \%$ with lipoma and only $46 \%$ with myelomeningocele showed clinical improvement postoperatively. Sixty two percent of the patients who presented with paraperesis improved following surgery while 37\% remained stable and only one patient deteriorated. Back and leg pain improved in $93 \%$ of patients and $50 \%$ of patients with urinary impairment improved.

Conclusions: Outcome of patients with TCS varies according to pathology and severity of symptoms. Diastematomyelia and thickened filum had the best outcome. The Karachi TCS severity scale is a valid tool for future studies.

Keywords: Spine; Tethered cord syndrome; Thickened filum; Outcome; Untethering

\section{Introduction}

Neural tube defects including the tethered cord syndrome (TCS) are still rising in developing countries like Pakistan, even though their prevalence has decreased worldwide. TCS is a stretch-induced functional disorder of the spinal cord with its caudal part anchored by an inelastic structure, which results in characteristic symptoms and signs [1]. This can be secondary to heterogeneous group of disorders, such as spinal lipomas, lipomatous filum, split cord malformations, and meningomyelocele. It typically occurs in children, and it is rare in adults [2]. The abnormally low position of the conus medullaris may lead to neurological, musculoskeletal, urological, orthopedic, or gastrointestinal abnormalities $[1,3]$. The clinical presentation of TCS is broad and varies with age at presentation as well as underlying cause. A physical examination has a key role in determining the diagnosis of TCS. Although TCS is a well-known entity, it continues to pose challenges regarding diagnosis and management [4]. In 1976, Hoffman

Received Nov 17, 2015; Revised Feb 9, 2016; Accepted Feb 10, 2016

Corresponding author: Salman Yousuf Sharif

Department of Spinal and Neurosurgery, Liaquat National Hospital, Karachi, 74800 Pakistan

Tel: +92-2134412464, Fax: +92-34140014, E-mail: sharifsalman73@gmail.com 
Table 1. Karachi tethered cord syndrome severity scale for clinical assessment [7]

\begin{tabular}{lllll} 
& \multicolumn{1}{c}{ Gait } & \multicolumn{1}{c}{ Power } & \multicolumn{1}{c}{ Bowel/urine } & Sensation \\
\hline 0 & Unable to walk & No contraction & Total incontinence & No sensation \\
1 & Severe Bilateral deficit & Flickering or trace of contraction & $\begin{array}{l}\text { Intermittent incontinence } \\
\text { uncontrolled }\end{array}$ & Diminished sensation \\
\hline 2 & Severe unilateral deficit & $\begin{array}{l}\text { Active movement with gravity } \\
\text { eliminated }\end{array}$ & $\begin{array}{l}\text { Intermittent incontinence, } \\
\text { controlled }\end{array}$ & Full sensation \\
\hline 3 & Mild bi or unilateral deficit & Active movement against gravity & Increased frequency & - \\
\hline 5 & Walks normally & Active movement against gravity & Total control & \\
\hline
\end{tabular}

et al. coined the term "tethered spinal cord" in patients with a low lying conus medullaris with a thickened filum [3]. More recently, there have been descriptions of TCS in which patients are described to have the conus medullaris in a normal position on imaging but presenting with signs and symptoms consistent with TCS [5]. Due to the variety of lesions and clinical presentations and the absence of high-quality clinical data, the decision regarding treatment is difficult [6]. The basic goal of surgical treatment in TCS is to improve or stabilize deficits in the symptomatic patient and to prevent future deficits in the asymptomatic patient [3].

Our objective was to look at the different presentations of TCS and their surgical outcome, according to the presenting complaints and pathologies.

\section{Materials and Methods}

We retrospectively reviewed 50 consecutive patients who were surgically treated for TCS at Liaquat National Hospital and Medical College, Karachi, from 2010 until 2014. Data was thoroughly reviewed and analyzed using IBM SPSS statistics ver. 21.0. Majority of our patients were between $0-15$ years of age with the mean age of 4 years. The follow-up period of these patients was 12-48 months. The patients were assessed with respect to power, gait, urinary and bowel control, and deformity. The presenting complaints, associated pathologies, and postoperative improvements were documented. The Karachi TCS severity scale for clinical assessment (Table 1) [7] was used to assess patients preoperatively, and the scores were compared to postoperative changes. The chi-square test was used to determine significant associations between variables at a $5 \%$ significance level.

\section{Surgical technique}

The surgical technique for division of thickened filum terminale is as follows. After induction of general anesthesia, the patient was positioned prone on two bolsters placed underneath the patient. The L5-S1 space (depending on the radiology, a space above was also used in some cases) was localized with fluoroscopy, reducing the injury of any low-lying sacral cell bodies. A small midline incision was made and dissection was extended down in a standard fashion to expose the inferior aspect of L-5, part or all of S-1, and S-1 laminectomy was performed. With microscopic guidance, a midline dorsal durotomy was performed and dural edges were held up with stitches. After opening the arachnoid, the filum could be recognized by typical dorsal midline location, bluish discoloration, anteriorly located vessels, and infiltrating fat (Figs. 1, 2). Intraoperative neurophysiological monitoring was used to distinguish functioning neural elements from the terminal filum. We did not have this facility at our institution and since we sectioned the filum at around the L5-S1 level in the majority of cases, monitoring was not essential. A suture was placed in the filum to send a specimen for pathology. We first coagulated and then sectioned the filum. A meticulous layered closure was done at the end.

\section{Results}

TCS patients ranged in age from $0-15$ years, with a mean of 4 years. Eighteen patients were 0-2 years of age and 25 were $2-15$ years of age. Of the 50 original patients, seven were lost during follow-up. The most common complaint (90\%) was urinary sphincter problems. Thirty three patients presented with back swelling or any cutaneous 


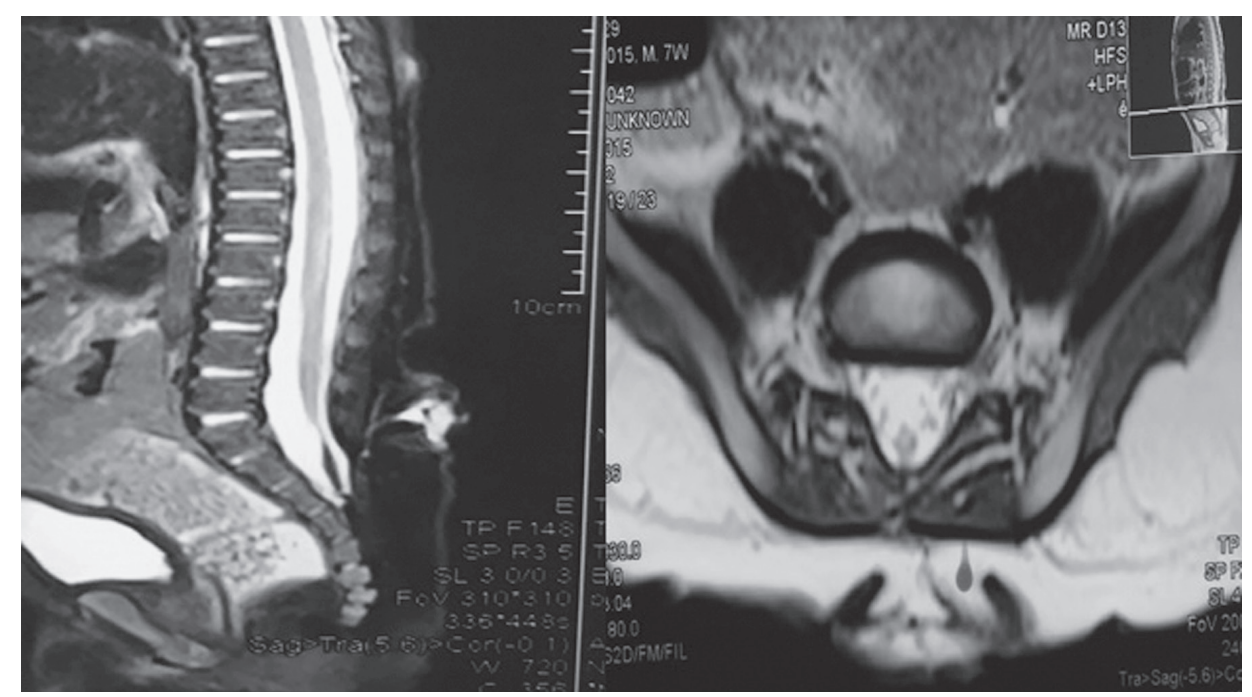

Fig. 1. Sagittal and axial T2 weighted magnetic resonance imaging of the dorsolumbar spine of a 6 month old baby, showing low lying conus and fatty filum tethered to $\$ 1-3$ with a large appendage going up to the dura.

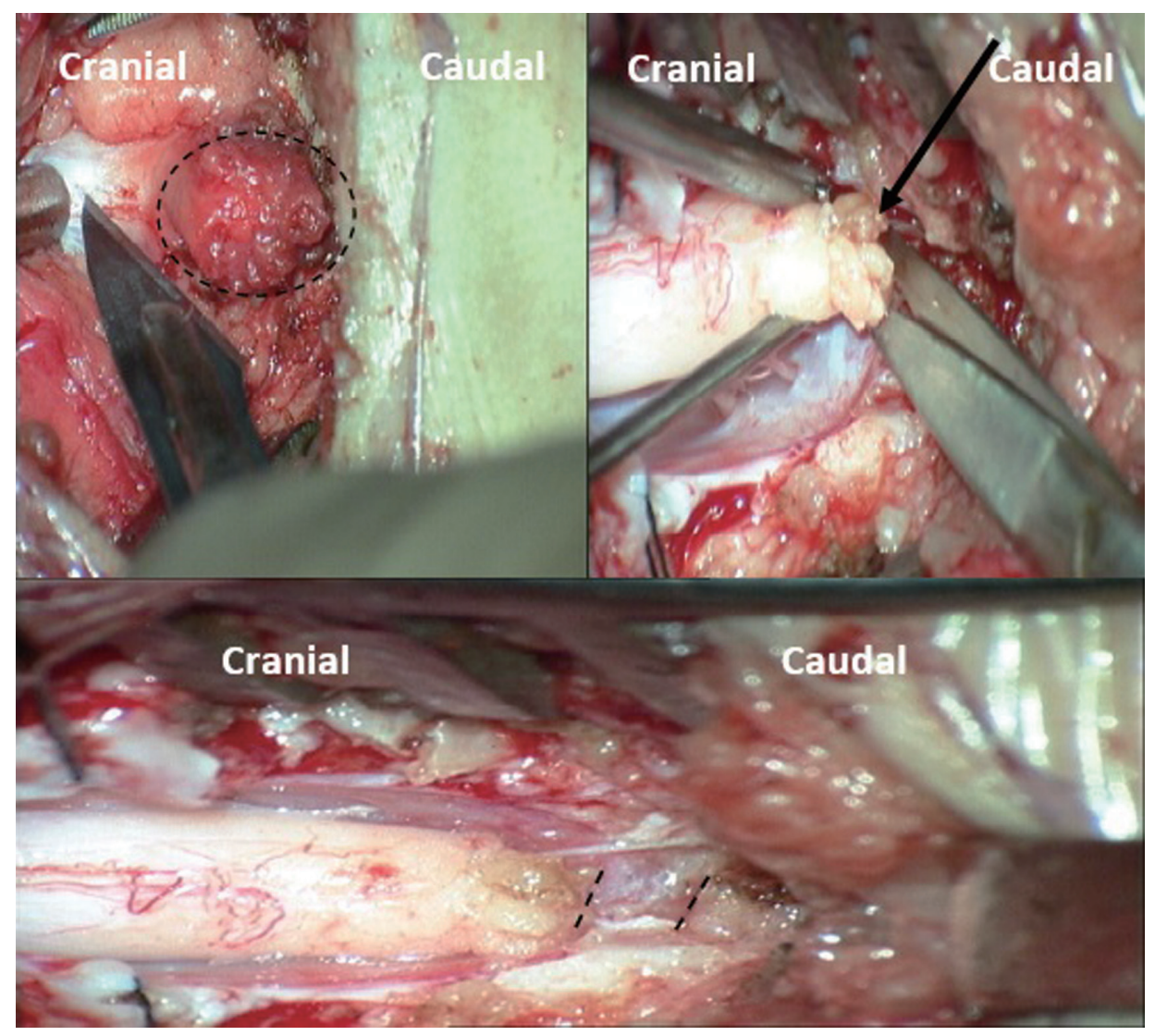

Fig. 2. Operative pictures of the same patient shown in Fig. 1. Base of appendage (black dotted circle) following opening of the dura. After coagulation of proximal and distal end of the filum, (arrow) sectioning resulting in proximal movement of the filum, revealing the empty space between two ends of untethered filum (divided ends are shown with black lines). 
symptom including lipomeningocele or previous myelomeningocele. Thirty two patients presented with progressive weakening of lower limbs. Twenty one patients had progressive scoliosis. Sixteen patients presented with progressive back or leg pain, with 14 patients presenting with Talipes equinovarus, seven with scissoring of feet and six patients were paraplegic. The presenting symptoms according to the age group are shown in Fig. 3

The majority of the patients had four distinct pathologies: thickened filum terminale, diastematomyelia, lipoma and myelomeningocele (Fig. 4). All patients had combinations of four pathologies (Fig. 5). Thirty patients had teth-

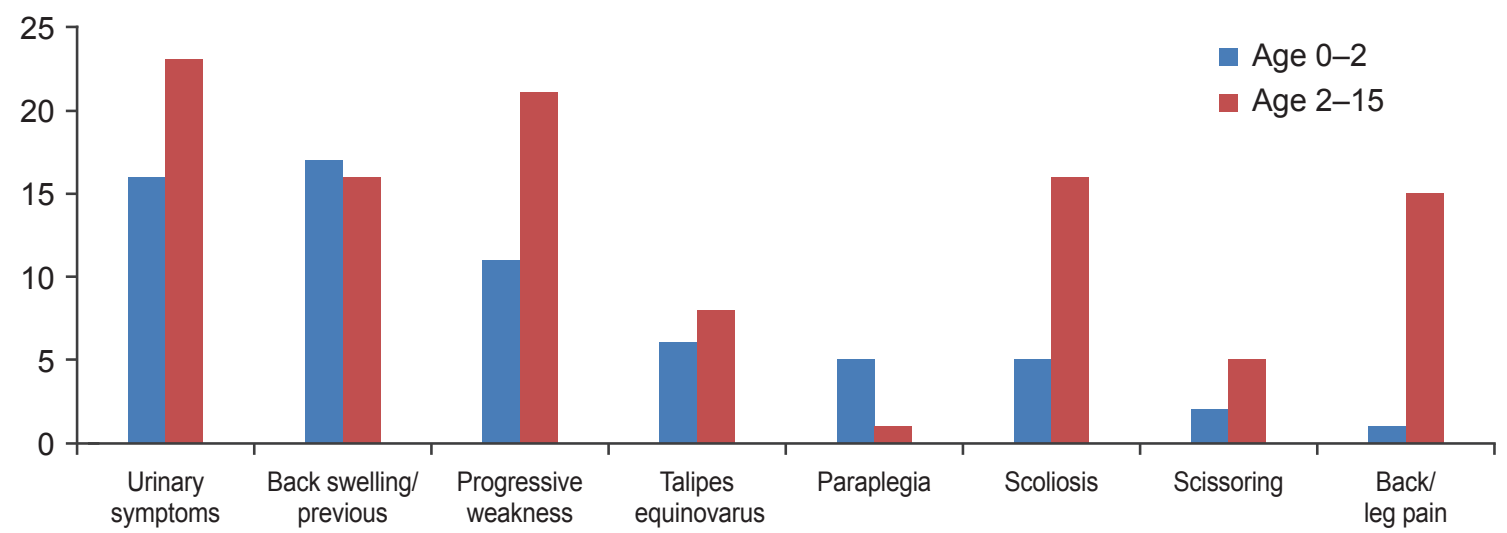

Fig. 3. Presenting complaints according to age group.
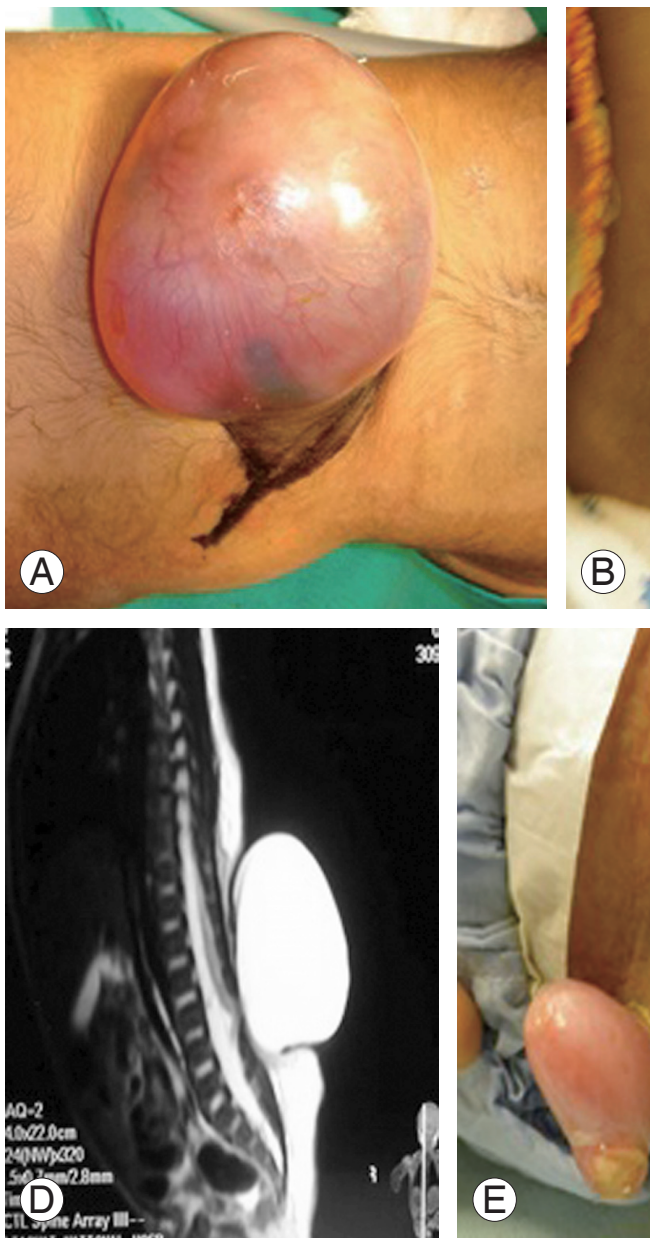

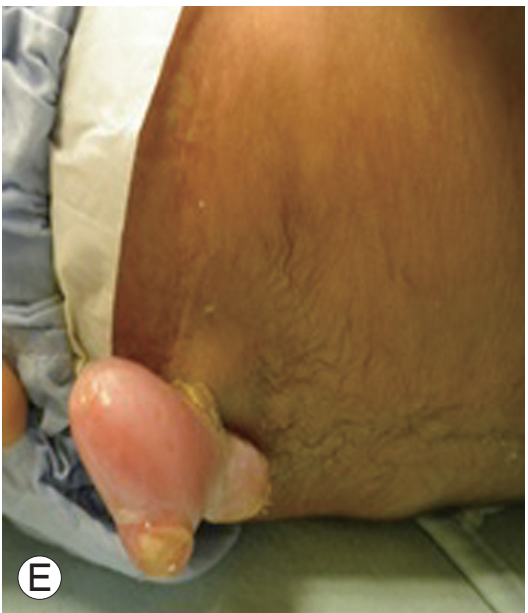

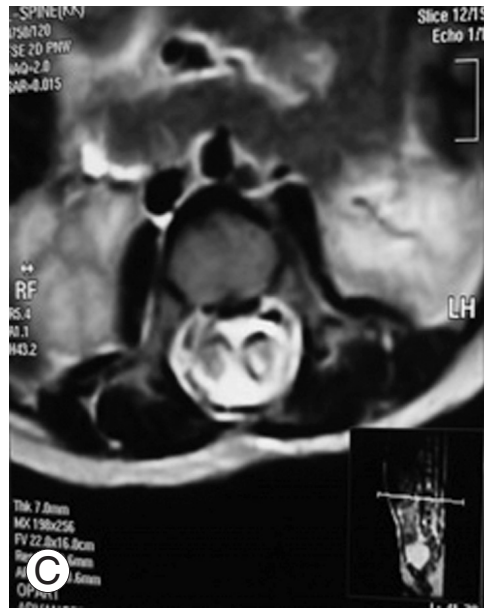

Fig. 4. (A) A large lumbosacral meningocele. (B) Hairy tuft with myelomeningocele in a year old child. (C) An axial image of T2 MRI of at L3 level showing diastematomyelia in a year old girl with TCS. (D) Sagittal view of T2 MRI of dorsolumbar spine showing a large lumbar meningocele with tethering at L4 level. (E) Garlic shaped meningocele with underlying tethering. MRI, magnetic resonance imaging; TCS, tethered cord syndrome. 
ering of the spinal cord at L3-S2 level that was apparent on resonance imaging (MRI). Five patients had tethering at L1-L2 and only one patient had tethering at the dorsal level.

The results of Karachi TCS severity scale for clinical assessment are shown in Fig. 6.

\section{Outcomes}

Eighty five percent of the patients with thickened filum terminale showed improvement postoperatively. Sixty six percent of the patients with diastematomyelia, $60 \%$ with lipoma and $46 \%$ with myelomeningocele improved

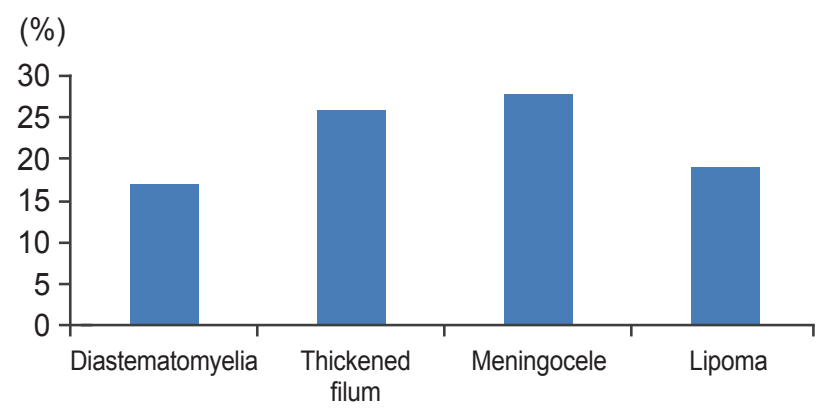

Fig. 5. Associated pathologies with tethered cord syndrome. postoperatively. Sixty two percent of the patients with paraperesis improved postoperatively, while $37 \%$ remained stable and only one patient deteriorated. Of the six patients with paraplegia, only one improved. Of the 15 patients that presented with back and leg pain, 93\% percent improved postoperatively. Of the 39 patients that presented with urinary impairment, $46 \%$ improved. Of the 38 patients with decreased power in lower limbs, $42 \%$ improved and 57\% stabilized postoperatively. The overall improvement according to the Karachi TCS Severity Scale is shown in Fig. 7

\section{Discussion}

Neural tube defects are still common in the developing world, due to lack of antenatal awareness and care $[8,9]$. In third world countries, TCS goes undiagnosed for years due to lack of tertiary care hospital care setups in rural areas. Although the incidence of spinal dysraphism has reduced [3], it is still significantly high in our part of the world because of the lack of awareness of risk factors $[8,9]$. The risks include folic acid deficiency [10], mothers very young or very old in age, toxic medications, obesity,
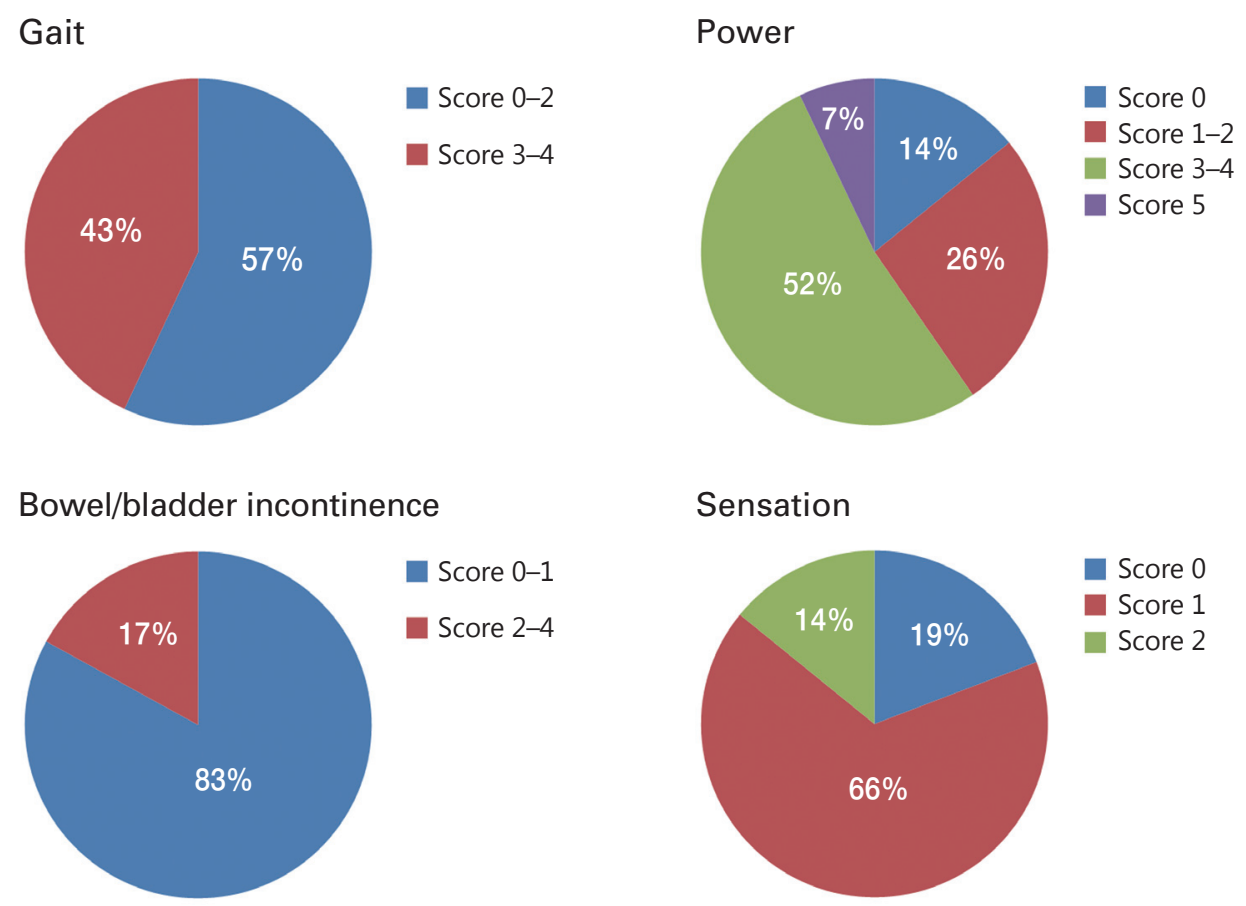

Fig. 6. Gait, power, bowel/bladder and sensation-the factors seen in Karachi TCS severity scale for clinical assessment. TCS, tethered cord syndrome. 
(\%)

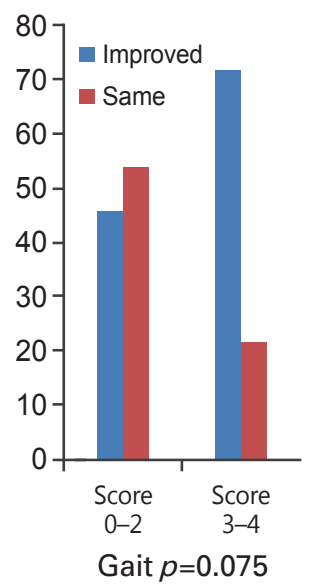

(\%)

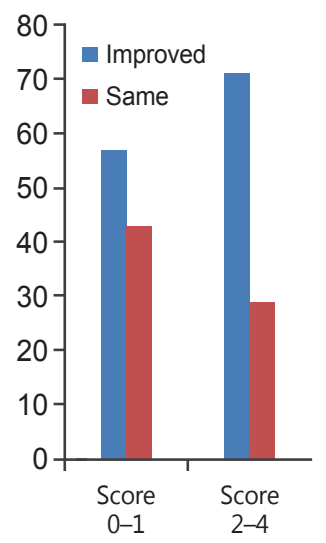

Bladder incontinence $p=0.67$
$(\%)$

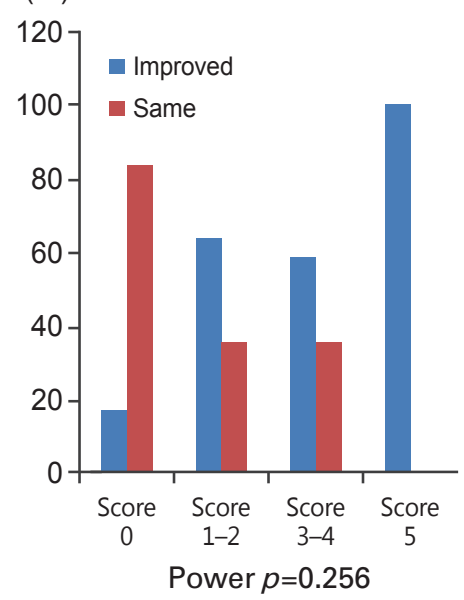

(\%)

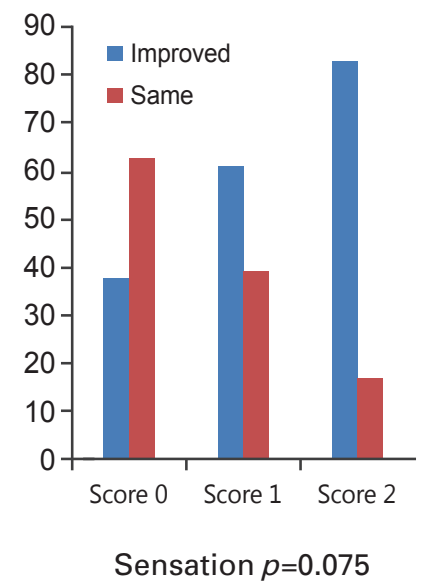

Fig. 7. Postoperative outcome of patients with Karachi TCS severity scale for clinical assessment. TCS, tethered cord syndrome.

multiple gestation, anti-epileptic medications, zinc deficiency, and ingestion of excessive tea in the first trimester of pregnancy [9].

Patients with TCS can present with a wide variety of signs and symptoms in combination with cutaneous, orthopedic, spinal, anorectal and urological abnormalities, as well as pain [3]. In our experience, the common presenting complaints were sphincter dysfunction, lower limb motor deficits, cutaneous signs at the lumbosacral region, and progressive leg pain. The other symptoms included, scoliosis, scissoring of feet, contractures, and Talipes equinovarus.

Urinary dysfunction is one of the most common finding in TCS. The use of the urodynamic test is helpful is diagnosing urinary dysfunction and assessing the patients pre and post operatively, especially in infants, in whom the clinical diagnosis of urinary dysfunction can be difficult [3]. Urinary abnormalities were the most common complaint found in patients $0-2$ years of age $(94 \%)$ and 2-15 years of age (95\%). The majority of our findings were based on patient recognition and parental assessment of their child. We were not able to use the urodynamic test as a definitive assessment and diagnostic tool because a lot of our patients were from a low socioeconomic background, which was a major drawback in our study.

The neurological findings in patients with TCS are varied, and include motor weakness, gait abnormality, spasticity, complete paraplegia, hyper or hyporeflexia, muscular atrophy, and sensory dysfunction [3]. In our study, more than $80 \%$ of the patients had neurological finding including paraperesis (74\%) and paraplegia (14\%). Motor function was easily assessed, documented, and compared postoperatively. But, there was a discrepancy in documented reflexes in our series, so reflexes were not included in our score.

The cutaneous findings in TCS usually include lipomas, hypertrichosis, hemangiomas, cigarette burns, dermal sinus tracts, and meningocele [3]. Cutaneous findings were evident in $76 \%$ of our patients, and the literature suggests that cutaneous manifestations are present in $70 \%$ of the patients with spinal dysraphism. Orthopedic deformities are also commonly found in patients with TCS, such as leg length discrepancy, (talipes equinovarus, TEV), contractures, club foot, progressive scoliosis, and scissoring of the legs. In our series of patients, the most common orthopedic deformity was scoliosis (48\%), followed by TEV (32\%) and foot deformities (16\%) (Fig. 8). In another study, scoliosis was documented in $25 \%$ of the cases and foot deformity was more commonly found [3]. Presently, scoliosis was more common among patients 2-15 years of age. Lower back pain and leg pain is more commonly found in adults with TCS [11].

TCS is usually associated with heterogeneous group of spinal dysraphism, such as diastematomyelia, lipoma, thickened filum, and myelomeningocele [12]. MRI is the modality of choice for evaluation of TCS [12] as it shows structural information required to assess, classify, and identify associated pathologies, and to plan treatment. Our patients had combinations of all four pathologies.

The surgical management of TCS remains controversial for many neurosurgeons. Patients with a significant abnormality, such as a lipomyelomeningocele and clear clinical 


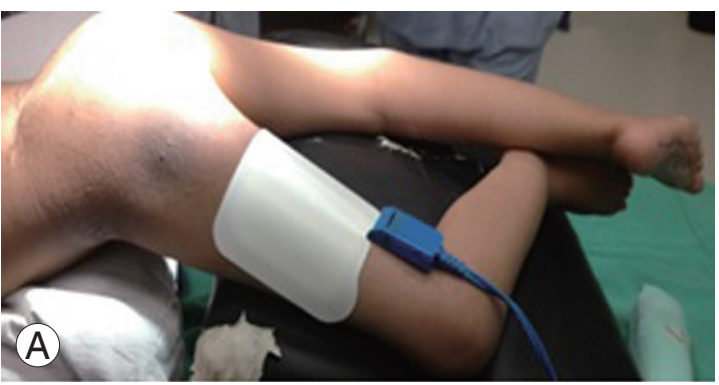

Fig. 8. (A) Scissoring of the legs along with talipes equinovarus before surgery for tethered cord in adolescent. (B) Kyphoscolisosis in a patient with tethered cord syndrome.

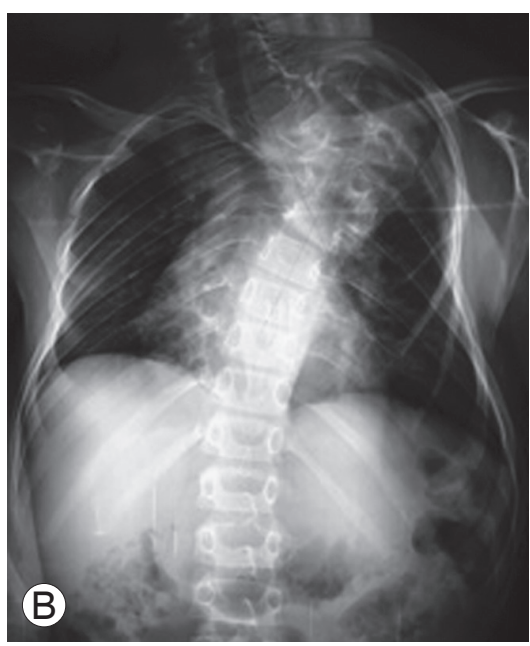

improvement postoperatively. Of the 19 patients who scored 3-4, 14 had improvement postoperatively. From the 17 patients who had TCS power score of $0-2$, only eight improved postoperatively. Of the 26 patients with a TCS power score of 3-5, 17 showed improvement postoperatively. Of the 35 patients who had TCS urinary/bowel score of $0-1,19$ showed improvement, and of the eight patients with a score of 3-4, six improved. Out of the eight patients with TCS sensation score of 0 , three improved. Finally, of the 35 patients with a score between 1-2, 24 showed improvement postoperatively.

The common postoperative complications of untethering include cerebrospinal fluid (CSF) leakage, wound infection, meningitis, bladder dysfunction, and neurological deterioration $[3,14]$. We encountered complications in seven patients, in which CSF leakage occurred in four patients, two patients had worsened urinary control, and postoperative infection. The CSF leak was surgically repaired in one of the patients, and the urinary control recovered in a patient over 6 months of follow-up. The patient with worsened urine control also had deterioration of left foot power postoperatively, but also recovered over next 2 months. The deterioration in neurology may have been because of the neural bands lying within the filum, which may have been sectioned, or because of manipulation and stretch of neural tissue during surgery.

Even though there is enormous data on TCS in children, there has yet to be a universal scale that can be applied to preoperative and postoperative assessment [15]. We modified a scale that was proposed on the basis of a clinical approach for TCS patients [7]. This includes gait, power, bowel/urine, and sensation. Gait was only applied when it

Of the 24 patients with TCS gait score of $0-2,11$ showed 
could be assessed.

The study has some limitations. Itis retrospective, although the final follow-up was prospective in all patients. Even with a short follow-up with small numbers, we have shown that thickened filum terminale patients had best outcome, followed by diastematomyelia and lipoma. We used the Karachi severity scale of TCS to score clinical symptoms. A prospective long\-term study with large numbers of patients is underway with application of the Karachi TCS severity scale.

Neural tube defects have a major impact on the health and quality of life of affected children and their families. Fortification of flour with folic acid has significantly reduced the number of children with neural tube defects in all countries that have mandated it [10]. The approach is recommended in developing countriesto reduce the incidence of neural tube defects.

\section{Conclusions}

TCS is a common condition in third world countries. It is essential to diagnose and surgically treat TCS in the early stage. Untethering in patients with TCS improves or arrests the progression of neurological deterioration. $\mathrm{Pa}$ tients with diastematomyelia and thickened filum presenting with TCS have better outcome compared to patients with myelomeningocele or meningocele. Our modified Karachi TCS severity scale for clinical assessment is good way to standardize the preoperative and postoperative clinical findings. A larger prospective study is already underway at our institution to explore the predictive value of the scale.

\section{Conflict of Interest}

No potential conflict of interest relevant to this article was reported.

\section{References}

1. Yamada S, Won DJ. What is the true tethered cord syndrome? Childs Nerv Syst 2007;23:371-5.

2. Shih P, Halpin RJ, Ganju A, Liu JC, Koski TR. Management of recurrent adult tethered cord syndrome. Neurosurg Focus 2010;29:E5.

3. Bui CJ, Tubbs RS, Oakes WJ. Tethered cord syndrome in children: a review. Neurosurg Focus 2007;23:E2.
4. Lee GY, Paradiso G, Tator CH, Gentili F, Massicotte EM, Fehlings MG. Surgical management of tethered cord syndrome in adults: indications, techniques, and long-term outcomes in 60 patients. J Neurosurg Spine 2006;4:123-31.

5. Hertzler DA 2nd, DePowell JJ, Stevenson CB, Mangano FT. Tethered cord syndrome: a review of the literature from embryology to adult presentation. Neurosurg Focus 2010;29:E1.

6. Drake JM. Surgical management of the tethered spinal cord: walking the fine line. Neurosurg Focus 2007;23:E4.

7. Eliyas MA. Tethered Cord Syndrome: neurophysiological monitoring in tethered cord syndrome [Internet]. Jordan: CNS Clinic; 2005 [cited 2016 Jul 5]. Available from: http://www.neurosurgery.tv/neurophysiologytetheredcord.html.

8. Khattak ST, Naheed T, Akhtar S, Jamal T. Incidence and risk factors for neural tube defects in Peshawar. Gomal J Med Sci 2008;6:1-4.

9. Hashim AS, Ahmed S, Jooma R. Management of myelomeningocele. J Surg Pakistan (Int) 2008;13:7-11.

10. Castillo-Lancellotti C, Tur JA, Uauy R. Impact of folic acid fortification of flour on neural tube defects: a systematic review. Public Health Nutr 2013;16:90111.

11. Kang JK, Yoon KJ, Ha SS, Lee IW, Jeun SS, Kang SG. Surgical management and outcome of tethered cord syndrome in school-aged children, adolescents, and young adults. J Korean Neurosurg Soc 2009;46:46871.

12. Halevi PD, Udayakumaran S, Ben-Sira L, Constantini S. The value of postoperative MR in tethered cord: a review of 140 cases. Childs Nerv Syst 2011;27:215962.

13. Sharif S, Allcutt D, Marks C, Brennan P. "Tethered cord syndrome": recent clinical experience. $\mathrm{Br} \mathrm{J} \mathrm{Neu}-$ rosurg 1997;11:49-51.

14. Balasubramaniam C, Rao SM, Subramaniam K. Management of CSF leak following spinal surgery. Childs Nerv Syst 2014;30:1543-7.

15. May L, Hayward R, Chakraborty A, et al. Lack of uniformity in the clinical assessment of children with lipomyelomeningocele: a review of the literature and recommendations for the future. Childs Nerv Syst 2013;29:961-70. 